\section{Kidney Blood Pressure Research}

Kidney Blood Press Res 2018;43:1034-1041 for commercial purposes as well as any distribution of modified material requires written permission.

\title{
Multiple Primary Malignancies in Renal Transplant Recipients: a Single Centre Retrospective Cohort Study
}

\author{
Nikolina Basic-Jukic ${ }^{\mathrm{a}}$ Jason Kirincich ${ }^{\mathrm{a}}$ Bojan Jelakovic ${ }^{\mathrm{a}}$ Zeljko Kastelan $^{\mathrm{b}}$ \\ aDepartment of Nephrology, Arterial Hypertension, Dialysis and Transplantation, University Hospital \\ Centre Zagreb, School of Medicine, University of Zagreb, Zagreb, 'bepartment of Urology, University \\ Hospital Centre Zagreb, School of Medicine, University of Zagreb, Zagreb, Croatia
}

\section{Key Words}

Renal transplantation • Malignant tumor • Multiple malignancies • Outcome

\begin{abstract}
Background/Aims: Renal transplant recipients are exposed to immunosuppressive treatment which may increase the risk for developing malignancies. Limited data exists concerning the occurrence of multiple primary malignancies (MPM) in renal transplant patients. Methods: All the patients who received a renal allograft at our institution from 1973 to 2017 were included in this investigation. Data from patients with more MPM were obtained from the charts and medical records. Malignancies were categorized as synchronous if the interval between occurrences was less than or equal to 6 months and metachronous if the interval was more than 6 months. Results: Out of the 1884 patients who received a renal allograft, 164 (8.7\%) developed a malignant tumor. Twenty-two patients (13.4\%; 6 females, 16 males) developed MPM, 7 synchronous (31.8\%) and 15 metachronous types (68.2\%). The most common initial primary tumors were skin cancers (8) and kidney cancers (3). Furthermore, skin cancers were the most common second primary malignancies (9). Log-rank analysis revealed significantly better survival in the synchronous group (113.3 months) than in the metachronous group (24.6 months) $(p=0.04)$. Conclusion: MPM are more frequent in renal transplant recipients than in the general population. It is associated with a high mortality rate, especially in the metachronous group. An increased awareness and frequent screening tests are necessary when managing this condition.




\section{Kidney Blood Pressure Research}

\section{Introduction}

Multiple primary malignancies (MPM) are a prevalent phenomenon that are usually defined as primary malignant tumors of different histological origins in one person [1]. They are defined by the following criteria: (1) every tumor has definite features of malignant change, (2) every tumor is anatomically separate, (3) the second tumor cannot be a metastatic lesion or due to recurrence of the first tumor, and (4) the following primary malignancies have to be present in either the same or different organs [2]. Multiple primary malignancies may be classified as synchronous or metachronous. The synchronous type is defined as malignancies that are observed at the same time or within 6 months of each other, and malignancies that develop after 6-months of one another are considered as metachronous MPM [3]. The etiology of this occurrence has not been completely elucidated. It is believed to involve the patient's exposure to known carcinogens like chemotherapy and radiotherapy during the treatment of the initial tumor, genomic structural variations that can increase a patient's tumorigenic susceptibility and the functional status of a patient's immune system [4].

Current literature contains limited data concerning MPM in renal transplant recipients. In this retrospective study, we analyzed the occurrence of MPM at our renal transplant program over a 44-year study period.

\section{Materials and Methods}

This was a retrospective cohort study including a series of 1884 consecutive renal transplant patients who received a renal allograft at the University hospital centre Zagreb from 1973 to 2017. Patients were regularly followed up every three months at the outpatient clinic. Immunosuppression has changed with time. Universal induction with basiliximab was introduced in 2006, and for sensitized patients we used polyclonal antibodies. Initial maintenance immunosuppressive therapy was usually done by utilizing a triple drug regimen containing cyclosporine (640 patients) or tacrolimus (1244 patients), azathioprine (482 patients) or mycophenolate (1402 patients) and steroids. From 1999 onward we generally used mycophenolate instead of azathioprine, and from 2007 onward tacrolimus instead of cyclosporine. After January 2008, patients with a history of malignancy or with post-transplant malignancy received an mTOR inhibitor instead of a calcineurin inhibitor if they had no contraindications to the medication (proteinuria, acute rejection, side-effects, or donor-specific antibodies) (92 patients). Acute rejection was recorded in 296 patients $(15.7 \%$ ). Episodes of acute rejection were treated with steroid pulses (296 patients), while 78 patients also underwent plasma exchange for treatment of humoral rejection, and 4 received ATG for treatment of steroid resistant acute rejection.

Patients were diagnosed based on medical history, physical examination, appropriate radiographic and/or endoscopic examinations, and pathological results. The time interval to differentiate between synchronous or metachronous types required 6 months. The inclusion criteria of patients in this study were the presence of at least two neoplastic locations confirmed by histopathological examination and tumors with distinct histopathology in different locations. Patients were excluded from the investigation if they didn't have a clear histopathological confirmation of each tumor, if the second tumor was suspected to be a metastasis of the first location, as well as patients with the first malignancy being diagnosed before transplantation and the second malignancy being diagnosed after the transplantation. Patient age at the time of each tumor diagnosis, gender, site of origin, synchronous or metachronous appearance, immunosuppressive protocol, treatment regimen and outcome have been recorded.

Statistical analysis was performed using Stata/SE 11.2 for Windows (StataCorp LP, USA). Differences between these two groups were analyzed by using Fisher's exact test or the Mann Whitney U test at the level of significance $\mathrm{P}<0.05$, with Kaplan-Meier analysis for survival.

The study protocol was approved by the Institutional Review Board and conformed to the ethical guidelines of the Declaration of Helsinki. 


\section{Kidney Blood Pressure Research}

\section{Results}

\section{Patients' characteristics}

Over the observed period, 1884 patients received a renal allograft (1114 male), with an average age at the transplantation of 55 years (range 18 to 77 years), and median followup time of 8 years. There were 673 transplantations from 1973 to 2007 and 1211 from 2007 to 2017, this abrupt increase can be attributed to Croatia entering the Eurotransplant organization during this time.

There were 164 patients who developed a malignancy, and 22 who had MPM. There was a male predominance ( 16 male vs. 6 female) and the median age at the time of diagnosis of the first primary malignancy was 61 (range 39-77) years. Primary kidney diseases included glomerulonephritis (7), endemic nephropathy (3), pyelonephritis (3), nephroangiosclerosis (2), diabetic nephropathy (1), polycystic kidney disease (1) and an idiopathic cause of kidney failure in 4 of the patients. The median time of dialysis vintage was 5.14 years (range 0.5-12 years). The time frame between transplantation, development of the first tumor and of the second tumor is shown in Table 1.

Four patients had two renal transplantations. Two patients had a history of malignancy before transplantation (one colon cancer and one basal cell carcinoma). Six patients were smokers. The immunosuppressive protocol included induction in 12 patients (basiliximab in 10 and antithymocyte globulin in only 2 patients). Sixteen patients received cyclosporine and 6 patients, tacrolimus. Azathioprine was used in 7, mycophenolate in 14 and everolimus in one patient. Two patients developed acute cellular rejection and were treated with steroid pulses.

Significantly more patients treated with cyclosporine or azathioprine developed MPM compared to patients treated with other immunosuppressive drugs (Fisher exact test, $\mathrm{p}=0.007$ ) (Table 2).

Three patients had chronic hepatitis $\mathrm{C}$ virus infections, and two had CMV infection. No other viral infections were recorded.

Synchronous and metachronous malignancies

Seven patients (32\%) developed synchronous and $15(68 \%)$ metachronous MPM. The mean time from renal transplantation until the diagnosis of MPM was 10 years (range 2-20) for the synchronous and 2 years (range 1-25) for the metachronous group (Mann-Whitney $\mathrm{p}=0.02$ ). Patients' characteristics based on the time of cancer occurrence are presented in Table 3.

There was no difference between the synchronous and the metachronous group regarding the type of primary kidney disease.

Table 3. Patients' characteristics based on the time of cancer occurrence are presented in Table 1. MPM, multiple primary malignancies; IQR, interquartile range). ${ }^{\dagger}$ Mann Whitney U test

\begin{tabular}{lcccc} 
Parameter & $\begin{array}{c}\text { Synchronous } \\
(\mathrm{n}=7)\end{array}$ & $\begin{array}{c}\text { Metachronous } \\
(\mathrm{n}=15)\end{array}$ & $\begin{array}{c}\text { Total } \\
(\mathrm{n}=22)\end{array}$ & $\mathrm{P}^{*}$ \\
\hline $\begin{array}{l}\text { Gender [n(\%)] } \\
\text { Male }\end{array}$ & $4(57)$ & $11(73)$ & $15(68)$ & 0,63 \\
$\quad 3(43)$ & $4(27)$ & $7(32)$ & \\
Female & $54(51-74)$ & $75(67-80)$ & $73(56-78)$ & $0,02^{\dagger}$ \\
Age [years] [Median (IQR)] & & & & \\
$\begin{array}{l}\text { Dialysis vintage [years] } \\
\text { [Median (IQR)] }\end{array}$ & $6(1-10)$ & $4(2-7)$ & $4,5(2-9)$ & $0,55^{\dagger}$ \\
\hline
\end{tabular}




\section{Kidney Blood Pressure Research}

Table 4. Localization and immunosuppression at the time of occurrence and treatment of the first primary malignancy. BCC, basal cell carcinoma; SCC, squamous cell carcinoma; C, cyclosporine; $\mathrm{T}$, tacrolimus; S, steroid; $\mathrm{M}$, mycophenolate; $\mathrm{A}$, azathioprine. *Fisher exact test

\begin{tabular}{|c|c|c|c|}
\hline \multirow[b]{2}{*}{ Primary malignancy } & \multicolumn{2}{|c|}{ Type } & \multirow[b]{2}{*}{$\mathrm{P}^{*}$} \\
\hline & $\begin{array}{c}\text { Synchronous } \\
(\mathrm{n}=7)\end{array}$ & $\begin{array}{c}\text { Metachronous } \\
(\mathrm{n}=15)\end{array}$ & \\
\hline \multicolumn{4}{|l|}{ First tumor [n(\%)] } \\
\hline $\begin{array}{l}\text { Kidney } \\
\text { adenocarcinoma }\end{array}$ & $2(29)$ & $1(7)$ & \multirow{10}{*}{0,39} \\
\hline BCC & $2(29)$ & $4(27)$ & \\
\hline Colon cancer & $1(14)$ & 0 & \\
\hline Urinary bladder & $1(14)$ & $1(7)$ & \\
\hline Thyroid & 0 & $3(20)$ & \\
\hline Prostatic gland & 0 & $2(13)$ & \\
\hline Ovarian & $1(14)$ & 0 & \\
\hline SCC & 0 & $2(13)$ & \\
\hline Merkel cell cancer & 0 & $1(6,7)$ & \\
\hline Breast & 0 & $1(6,7)$ & \\
\hline \multicolumn{4}{|l|}{ Immunosuppression 1 [n(\%)] } \\
\hline $\mathrm{C}+\mathrm{M}+\mathrm{S}$ & $3(43)$ & $6(40)$ & \multirow{4}{*}{$>0,99$} \\
\hline $\mathrm{C}+\mathrm{A}+\mathrm{S}$ & $2(29)$ & $5(33)$ & \\
\hline $\mathrm{T}+\mathrm{M}+\mathrm{S}$ & $2(29)$ & $3(20)$ & \\
\hline $\mathrm{mTOR}+\mathrm{T}+\mathrm{S}$ & 0 & $1(7)$ & \\
\hline \multicolumn{4}{|l|}{ Treatment $1[\mathrm{n}(\%)]$} \\
\hline Operative & $6(86)$ & $8(53)$ & \multirow{5}{*}{0,51} \\
\hline Operative + mTOR & $1(14)$ & $1(7)$ & \\
\hline Operative + irradiation & 0 & $4(27)$ & \\
\hline $\begin{array}{l}\text { Operative }+ \\
\text { radioactive iodine }\end{array}$ & 0 & $1(7)$ & \\
\hline Operative + tamoxifen & 0 & $1(7)$ & \\
\hline
\end{tabular}

Table 5. Localization and immunosuppression at the time of occurrence and treatment of the second primary malignancy. BCC, basal cell carcinoma; SCC, squamous cell carcinoma; C, cyclosporine; T, tacrolimus; S, steroid; $\mathrm{M}$, mycophenolate; A, azathioprine. *Fisher exact test

\begin{tabular}{lccc}
\hline \multirow{2}{*}{ Secondary tumor } & \multicolumn{3}{c}{ Type } \\
& $\begin{array}{c}\text { Synchronous } \\
(\mathrm{n}=7)\end{array}$ & $\begin{array}{c}\text { Metachronous } \\
(\mathrm{n}=15)\end{array}$ & $\mathrm{P}^{*}$ \\
\hline Tumor 2 [n(\%)] & & & \\
Kidney & $1(14)$ & $1(7)$ & \\
BCC & $1(14)$ & $3(20)$ & \\
Colon & $3(43)$ & 0 & \\
Urinary bladder & 0 & $2(13)$ & \\
Ovary & $1(14)$ & 0 & 0,27 \\
SCC & $1(14)$ & $4(27)$ & \\
Breast & 0 & $1(7)$ & \\
Lung & 0 & $1(7)$ & \\
Plasmocytoma & 0 & $1(6,7)$ & \\
Glyoblastoma & 0 & $1(6,7)$ & \\
Melanoma & 0 & $1(6,7)$ & \\
C+M+S & & & \\
mTOR + M + S & $1(17)$ & 0 & \\
T + M + S & $3(50)$ & $11(73)$ & 0,26 \\
C + S & $2(33)$ & $2(13)$ & \\
Immunosuppression 2 [n(\%)] & 0 & $2(13)$ & \\
Operative & & & \\
Operative + mTOR & $2(29)$ & $12(80)$ & \multirow{2}{*}{0,07} \\
Palliative & 0 & $3(20)$ & \\
\hline & & &
\end{tabular}

Localization and immunosuppression at the time of occurrence and treatment of the first primary malignancy are presented in Table 4 . The most common first primary malignancy was basal cell carcinoma in $27 \%$ of cases.

Localization and immunosuppression at the time of occurrence and treatment of the primary malignancy is presented in Table 5 . The most common second primary carcinoma was squamous cell carcinoma of the skin, occurring in $23 \%$ of patients.

Tumor correlations for synchronous tumors were: colon-colon, kidney-colon, ovarycolon, urinary bladder-ovary, kidney-kidney, skin SCC- skin BCC, skin BCC- skin SCC.

Tumor correlations for metachronous tumors were: thyroid-lung, BCC-SCC (2 patients), SCC-BCC ( 2 patients), Merkel cell skin cancer-SCC, prostate-urinary bladder, urinary bladderkidney, kidney-urinary bladder, prostate-SCC, BCC-plasmocytoma, BCC-glyoblastoma, thyroid-melanoma, breast-BCC, thyroid-breast.

Log-rank analysis revealed significantly better survival in the synchronous group (113, 3 months, 95\% CI 79, $3-147,2)$ than in the metachronous group $(24,6$ months, $95 \%$ CI 14.7-34.5) (p=0.04) (Fig. 1).

\section{Discussion}

The frequency of MPM ranges between $2.4 \%$ and $20 \%$ based on the definition in literature and is increasing with longer patient follow-up [5]. The incidence of MPM has increased significantly due to improvements in diagnostic methods and with the discovery of new treatments, which allows for more patients to survive long enough to develop additional primary tumors [6]. Attributing factors involve improved screening, advances in diagnostic testing, more sophisticated anticancer treatment regimens, an improved overall surveillance of patients with cancer and better nutritional support. Cancer survivors may be more susceptible to developing second primary malignancies due to many factors which include a genetic predisposition towards cancer, environmental exposures, specific 


\section{Kidney Blood Pressure Research}

characteristics of some tumors, and adverse effects of treatment with chemotherapy and/or radiotherapy. Increased risk for MPM was found to correlate with family history, race, younger age at the time of diagnosis, a lower tumor stage and with indolent cancer behavior which enables prolonged survival [7]. Although there are numerous reports addressing the clinical features of MPM in the general population, literature on this topic in the renal transplant population is scarce.

Therefore,

this retrospective study was

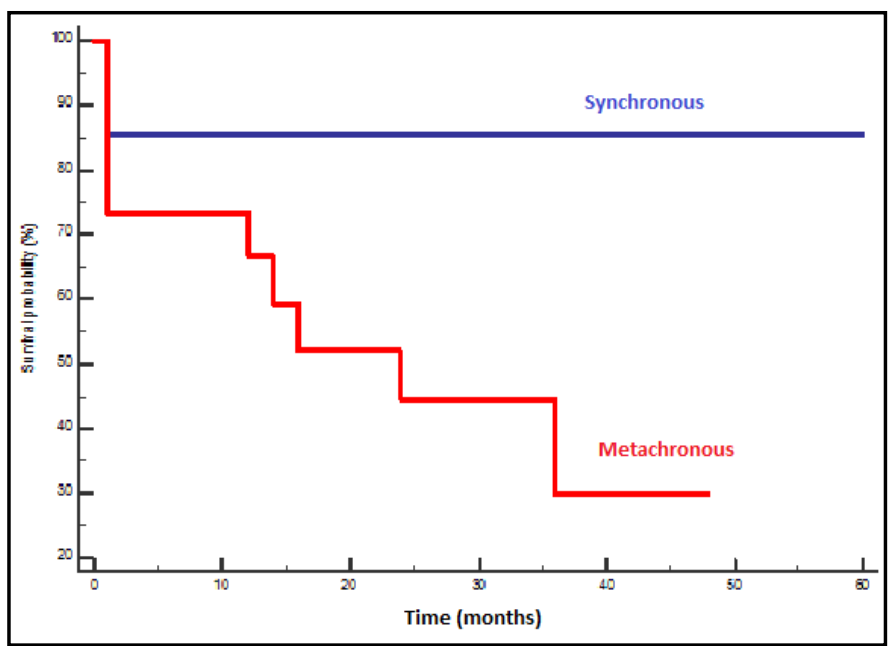

Fig. 1. Kaplan-Meier survival analysis comparing survival of patients with synchronous and metachronous multiple primary malignancies.

designed to evaluate the clinical

characteristics and outcomes of MPM involving renal transplant patients. The incidence of MPM in our study was $1.16 \%$ among all renal transplant recipients and $13.4 \%$ among renal transplant patients with malignancies. Of the 22 cases ( 6 females, 16 males) diagnosed with MPM, 7 were synchronous (31.8\%) and 15 were the metachronous variant (68.2\%). In the study of Bisof et al. from our institution, the incidence of MPM in the general population of oncology patients was $2.4 \%$. Among 103 patients, 83 cases were metachronous $(80.5 \%)$, and 20 cases (19.5\%) were synchronous malignancies. The frequency was higher in females than males. The most common tumor combinations in males were: prostate cancer-digestive system malignancy (especially colorectal cancer) and vice versa, and hematological malignant tumors-digestive system malignancy. While in women the most frequent were: breast cancercancer of contralateral breast and hematological malignant tumors (especially lymphoma non Hodgkin)-breast cancer [8]. In the transplanted population from our institution, the most common tumor combinations were different skin malignancies. Based on this comparison, it is obvious that the incidence of MPM was higher in renal transplant recipients (13.4\%) than in the general population (2.4\%). Additionally, in the general population MPM was more common in female and renal transplant populations than in male patients, with a diverse spectrum of localizations.

Our results are in contrast with previously reported data. Santangelo et al. did not find a higher risk for development of MPMs as compared to the corresponding general population. Their explanation is based on the fact that kidney-transplanted patients probably die before a new "second primary malignancy" appears or due to interruptions of immunosuppressive therapy, thus limiting the exposure to oncogenic effects of such drugs over time while they return to dialysis. In their practice, it was hard to find transplanted patients who survived the first malignancy and then continued immunosuppressive treatment long enough to develop a second primary cancer as a consequence of iatrogenic immune deficiency [9]. Taioli et al. investigated the incidence of a MPM in 7,636 patients who underwent a kidney, liver, lung or heart transplant between 1970 and 2004. They did not find an increased risk of MPM in transplanted patients with a previous malignancy [10]. To our knowledge, only these two reports have specifically examined the incidence of MPMs in transplanted patients. Tessari et al. reported an increased risk for the development of primary and second cancers in the renal transplant population, with the type of second cancer being dependent on the type of the primary. Patients with nonmelanoma skin cancer developed a second nonmelanoma skin cancer, and patients with nonskin cancer developed a second nonskin cancer [11]. Our 


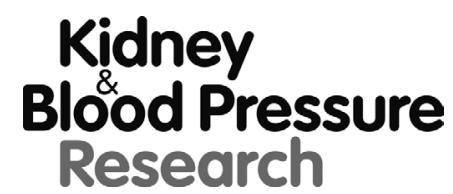

Kidney Blood Press Res 2018;43:1034-1041
\begin{tabular}{l|l} 
DOI: 10.1159/000490825 & (c) 2018 The Author(s). Published by S. Karger AG, Basel \\
Published online: 25 June, 2018 & www.kargercom/kbr
\end{tabular}

Basic-Jukic et al.: Multiple Primary Malignancies After Renal Transplantation

results demonstrate that all types of tumors may occur in various possible combinations, and are not limited to skin or nonskin localizations.

Our results may differ from the results of Santangelo [9] and Taioli [10] for the following reasons: after malignancy diagnosis our patients are allowed (after detailed information) to choose between three different approaches: cessation of immunosuppression and graftectomy, minimization of existent immunosuppressive therapy or switching to an mTOR based immunosuppressive regimen (instead of the calcineurin inhibitor). So far, none of the patients have decided to stop immunosuppression and return to dialysis. Additionally, an exponential increase in the number of transplantations performed in Croatia has enabled transplantations for very old patients who are prone to develop malignancies. Based on a combination of factors (improved diagnosis, improved treatment, demographics), it may be expected that this aging transplant population will lead to an increased prevalence of MPM.

Our study observed a higher number of the metachronous subtype versus synchronous malignancies. This contrasts the results of Santangelo et al. but is in agreement with a large observational study $[9,12]$. While our results show a significant mortality advantage of synchronous over metachronous malignancies, the observational study revealed the opposite with a median survival time that was greater for the metachronous subtype, 96 months from diagnosis compared to 12 months. Their study showed that an interval time of less than 60 months was a positive prognostic factor for patients with metachronous cancer [12].

In contrast to the healthy population, squamous cell carcinoma of the skin is more common than basal cell carcinoma in immunosuppressed patients. However the limited number of skin cancer occurrences in this study limits the support of this conclusion in renal transplant recipients with MPM [9].

Survival of patients with MPM in the general population depends on the cancer type and stage at diagnosis, but also on comorbidities, behavioral and genetic factors. In renal transplant recipients, allograft dysfunction can cause lymphocyte attenuation and the immunosuppression may affect survival by altering the immune response. This along with immunosuppressive therapy can have direct oncogenic effects by inhibiting apoptosis, increasing the expression of transforming growth factor-beta (TGF-beta) and vascular endothelial growth factor (VEGF) (cyclosporine and tacrolimus), inducing DNA damage and negatively influencing DNA repair (azathioprine), or by promoting genetic mutations (polyclonal antibodies). On the contrary, it has been shown that some tumor types may regress with the use of mTOR inhibitors [13] and mycophenolate [14]. In our cohort, we did not find any difference between the type of immunosuppression and time-frame of cancer occurrence, or with survival. However, a significantly higher proportion of patients treated with cyclosporine or with azathioprine developed MPM than those who received tacrolimus, mycophenolate or everolimus. The small number of patients limits further statistical analysis to reveal the role of prolonged exposure to immunosuppression in general (which is present in patients who receive cyclosporine or/and azathioprine).

Renal transplant patients who are smokers, alcoholics, have a hereditary predisposition or with a primary malignancy before transplantation have an increased risk for the development of MPM. Additionally, the number of octogenarians receiving allografts is increasing. All these factors may contribute to the problem of MPM in the renal transplant population. As a preventive strategy, renal transplant recipients should be encouraged to stop the use of alcohol and tobacco, exercise regularly and adopt a healthy diet in order to regulate body weight. Regular dermatological examinations are mandatory. Studies have shown that the immunocompromised state of dialysis patients leads to a significant increase in malignancies due to uremic and dialysis prompted lymphocyte dysfunction, and several metabolic misbalances. However, there has not been a comprehensive study of MPM in this population [15].

There are some limitations of our study. During the observed period, the changing landscape of recipient demographics and comorbidities may influence our results. For 


\section{Kidney Bloód Pressure Research}

different reasons many patients have changed their initial immunosuppressive protocol during the post-transplant follow-up. The small number of patients on T cell depleting agents and with viral infections prevent an analysis about their possible roles in oncogenesis, which are well known drivers of post-transplant neoplasia [15].

\section{Conclusion}

Multiple primary malignancies were not uncommon in our cohort and occurred synchronously or metachronously. With regular follow-up of patients with a primary malignancy, most of the metachronous second primary malignancies could be detected at an earlier stage.

\section{Acknowlegdements}

The authors contributed to this paper in the following: Nikolina Basic-Jukic: design, performed research, wrote the paper; Jason Kirincich: collected data, wrote the paper; Bojan Jelakovic: data analysis, final approval; Zeljko Kastelan: performed research, wrote the paper.

\section{Disclosure Statement}

We have no conflicts of interest.

\section{References}

1 Coleman MP: Multiple primary malignant neoplasms in England and Wales, 1971-1981. Yale J Biol Med 1986;59:517-531.

2 Warren S, Gates O: Multiple primary malignant tumors: a survey of the literature and statistical study. Am J Cancer 1932;16:1358-1414.

3 Moertel CG, Dockerty MB, Baggenstoss AH: Multiple primary malignant neoplasms. I. Introduction and presentation of data. Cancer 1961;14:221-230.

4 Escobar PA, Smith MT, Vasishta A, Hubbard AE, Zhang L: Leukaemia-specific chromosome damage detected by comet with fluorescence in situ hybridization (comet-FISH). Mutagenesis 2007;22:321-327.

5 Hu NC, Hsieh SC, Chen TJ, Chang JY: Multiple primary malignancies including colon, stomach, lung, breast and liver cancer: a case report and literature review. Chinese Med J 2009;122;24:3091-3093.

6 Vogt A, Schmid S, Heinimann K, Frick H, Herrmann C, Cerny T, Omlin A: Multiple primary tumours: challenges and approaches, a review. ESMO Open 2017;2:e000172.

7 Wood ME, Vogel V, Ng A, Foxhall L, Goodwin P, Travis LB: Second malignant neoplasms: Assessment and strategies for risk reduction. J Clin Oncol 2012;30:3734-3745.

-8 Bisof V, Basic-Koretic M, Juretic A, Krpan AM, Samardic KR, Rakusic Z, Santek F, Krajina Z, Podolski P, Saric N, Crnjevic TB: Multiple primary malignancies. Lijec Vjesn 2011;133:384-388.

-9 Santangelo ML, Criscitiello C, Renda A, Federico S, Curigliano G, Dodaro C, Scotti A, Tammaro V, Calogero A, Riccio E, Pisani A, Carlomagno N: Immunosuppression and Multiple Primary Malignancies in KidneyTransplanted Patients: A Single-Institute Study. Biomed Res Int 2015;2015:183523.

10 Taioli E, Piselli P, Arbustini E, Boschiero L, Burra P, Busnach G, Caldara R, Citterio F, De Juli E, Dissegna D, Gotti E, Marchini F, Maresca MC, Marsano L, Montagnino G, Montanaro D, Sandrini S, Pedotti P, Scalamogna M, Serraino D: Incidence of second primary cancer in transplanted patients. Transplantation 2006;81:982985. 


\section{Kidney

Basic-Jukic et al.: Multiple Primary Malignancies After Renal Transplantation

11 Tessari G, Naldi L, Boschiero L, Minetti E, Sandrini S, Nacchia F, Valerio F, Rugiu C, Sassi F, Gotti E, Fonte L, Talamini G, Girolomoni G: Incidence of primary and second cancers in renal transplant recipients: a multicenter cohort study. Am J Transplant 2013;13:214-221.

12 Lv M, Zhang X, Shen Y, Wang F, Yang J, Wang, B, Chen Z, Li P, Zhang X, Li S, Yang J: Clinical analysis and prognosis of synchronous and metachronous multiple primary malignant tumors. Medicine 2017;96:6799.

$\$ 13$ Gaumann A, Schlitt HJ, Geissler EK: Immunosuppression and tumor development in organ transplant recipients: the emerging dualistic role of rapamycin. Transplant Int 2008;21:207-217.

14 Robson R, Cecka JM, Opelz G, Budde M, Sacks S: Prospective registry-based observational cohort study of the long-term risk of malignancies in renal transplant patients treated with mycophenolate mofetil. Am J Transplant 2005;5:2954-2960.

15 Vajdic CM, McDonald SP, McCredie MR, van Leeuwen MT, Stewart JH, Law M, Chapman JR, Webster AC, Kaldor JM, Grulich AE: Cancer incidence before and after kidney transplantation. JAMA 2006;296:28232831. 